\title{
La crianza de cerdos en vida libre y pecaríes silvestres en zonas de transición de áreas protegidas del sureste de México
}

\author{
Sanvicente, L.M.; Vargas, L.S. ${ }^{\circledR}$; Bustamante, G.A. y Jaramillo, V.J.L.
}

Colegio de Postgraduados. Puebla. Puebla. México.

\section{PALABRAS CLAVE}

Cerdos criollos.

Pecaries.

Itinerancia.

Apacentar.

Calakmul.

\section{RESUMEN}

El estudio analizó los sistemas de producción de cerdos domésticos en vida libre y pecaríes sitvestres para determinar los beneficios sociales, económicos y ambientales en comunidades ubicadas en la zona de transición de la Reserva de la Biósfera Calakmul, México. La información se obtuvo de 169 campesinos criadores de suinos, de un censo realizado en 13 comunidades. La información de la unidad de producción, los criadores, la piara de cerdos, la alimentación, los parámetros productivos y reproductivos, la comercialización, los costos e ingresos se registraron en un cuestionario. Los datos se analizaron con frecuencias, análisis de varianza, clúster, factorial y componentes principales con el paquete estadístico SAS. Se identificaron tres sistemas de crianza de cerdos domésticos en vida libre: traspatio $(23,1 \%)$, itinerancia estacional $(21,9 \%)$ e itinerante $(55 \%)$. Los tres sistemas tuvieron pecarí de collar (Pecarí tajacu) como una forma de reproducción en cautiverio de esta especie silvestre. El sistema de crianza en traspatio fue el más intensivo en uso de mano de obra, forraje de corte, grano de maíz y la compra de alimento. El sistema de itinerancia estacional fue intermedio en el uso de recursos e insumos. La crianza itinerante tuvo mayor convivencia con el pecarí en cautiverio, con el uso de los ecosistemas naturales y una menor orientación al mercado. La crianza en traspatio y la itinerancia estacional se clasificaron como de transición a la producción en confinamiento, con una disminución de la crianza en libertad y se incrementó el uso de insumos para la alimentación.

\section{The free ranging domestic pigs and wild peccaries raising in buffer zones of protected areas in southeastern Mexico}

\begin{abstract}
SUMMARY
The study analyzed the raising of domestic free ranging pigs and wild peccaries in order to determine the social, economic and environmental benefits in communities located in the transition zone of the Calakmul Biosphere Reserve, Mexico. The information was obtained from 169 farmers involved in pig keeping from a census conducted in 13 communities. The information of the production unit, the pig herd, the feeding, the productive and reproductive parameters, the commercialization, the costs and income was recorded in a questionnaire. The data were analyzed with frequencies, analysis of variance, cluster, factorial and principal components with the SAS statistical package. Three systems of productions of domestic pigs in freerange were identified: backyard pigs raising $(23,1 \%)$, seasonal roaming pigs $(21,9 \%)$, and free roaming pigs $(55 \%)$. The three systems had the collared peccary (Pecari tajacu) as a reproduction form in captivity of this wildlife species. The backyard pig raising was the most intensive in labor force, cutting forage, maize and feed purchase. The seasonal roaming pigs was evaluated as intermediate in the use of resources and supplies. The free roaming pigs was the one with the highest relation with the captive collared peccary breeding, and uses the natural ecosystems and it was not market oriented. The backyard and seasonal roaming pigs were classified like transition towards the stalf feed pigs, free ranging decreased and the use of external supplies increased.
\end{abstract}

\section{INTRODUCCIÓN}

Los sistemas de producción de cerdos en vida libre más comunes son la crianza en campo y de traspatio (Reynolds et al. 2015; Thys et al. 2016). El nivel de itinerancia de los cerdos en estos sistemas depende de la cantidad de insumos disponibles para la alimentación, el nivel socioeconómico de la familia, la infraestructura y la disponibilidad de mano de obra. En este sentido, la crianza de cerdos en vida libre es diversa, desde aquellas en donde las familias son de escasos recursos, viven en áreas marginadas y con áreas de bosques has- ta los sistemas con uso de tecnología y mano de obra más intensiva (Leluke y Kyvsgaard 2003; Dione et al. 2014). En condiciones de pequeña escala, los cerdos son confinados una parte del año, cuando hay escasez de alimentos o durante el crecimiento de los cultivos (Dione et al. 2014), encierre durante la noche y se dejan en libertad en el día para evitar la pérdida de animales (Thomas et al. 2013) o bien se ata al cerdo con una soga en los terrenos para que se alimente (Thomas et al. 2013; Reynolds et al. 2015). 
Por la finalidad productiva, en la crianza de cerdos en vida libre destacan diferentes propósitos: la engorda en agroecosistemas de dehesas (Edwards et al. 2005; Rodríguez-Estévez et al. 2009; Hadjikoumis 2012), la crianza en traspatio y en campo (Lemke et al. 2006; Phengsavanh et al. 2011; Leslie et al. 2015) y la crianza en áreas naturales protegidas (Rosenthal 2010; Karki 2013; Thys et al. 2016; Levin y Ayres 2017). Los estudios en las áreas naturales protegidas valoraron los beneficios sociales y ambientales de la crianza de cerdos, como es la contribución a la dispersión de semillas y la creación de nichos para el desarrollo de especies de plantas (Rosenthal 2010); por consumir plantas silvestres, los productos del cerdo pueden tener un beneficio funcional para los humanos (Ahmed et al. 2016).

En el caso de México, no hay estudios de la crianza de cerdos domésticos y su convivencia con los pecaríes silvestres en cautiverio en las comunidades usuarias de áreas naturales protegidas, aun cuando el propósito de estas áreas es vincular la conservación de la biodiversidad con las actividades humanas (Manson 2006; Ruiz-Mallén et al. 2015). Una de las especies de suinos silvestre que se ha criado en cautiverio es el pecarí de collar (Borges-Ventura et al. 2014), el cual es común en las áreas de selvas tropicales y muy ligado a la cultura de las comunidades rurales, como fue citado para la región Amazónica (Navarrete et al. 2000). Además de la crianza en cautiverio, el pecarí de collar tiene la posibilidad de manejarse en grupos numerosos y en grandes superficies, lo cual puede reducir los costos de alimentación e infraestructura (Jori et al. 2014); sobre todo, porque la dieta es a base de las partes vegetativas y reproductivas de las plantas (Kiltie 1981). En cautiverio, el pecarí de collar tiene un incremento rápido de las poblaciones y esto permite el manejo de grandes grupos en libertad o encierre estacional en los ecosistemas naturales (Jori et al. 2014).

Una de las áreas tropicales protegidas más importante en México es la Reserva de la Biósfera Calakmul, en el sureste del país, que es una zona de bosque tropical, con una mezcla de selva alta, mediana, baja y vegetación acuática. El Programa de manejo de la Reserva establece que la actividad ganadera tiene una mayor presión sobre la vegetación; asimismo, plantea que para recuperar y proteger la diversidad biológica se deben de establecer criaderos con especies silvestres y de fácil manejo, en lugar de los animales domésticos, como alternativas para la recuperación de la biodiversidad y el abastecimiento de proteína de origen animal para los habitantes del área (Instituto Nacional de Ecología 2000). De acuerdo a lo expresado, el objetivo del presente estudio fue analizar los sistemas de producción de cerdos domésticos en vida libre y pecaríes silvestres para determinar los beneficios sociales, económicos y ambientales en las comunidades ubicadas en la zona de amortiguamiento de la Reserva de la Biósfera Calakmul, México.

\section{MATERIAL Y MÉTODOS}

\section{ÁREA DE ESTUDIO}

El estudio se realizó en comunidades rurales ubicadas en la zona de transición de la Reserva de la
Biósfera Calakmul $\left(1^{\circ} 15^{\prime}\right.$ y $17^{\circ} 45^{\prime} \mathrm{N}, 90^{\circ} 10^{\prime}$ y $89^{\circ} 15^{\prime}$ W), estado de Campeche, en el sureste de México. La altitud es de 0 a $360 \mathrm{msnm}$. El clima es cálido húmedo con abundantes lluvias en verano (Am (f)), con precipitación media anual de $1300 \mathrm{~mm}$, con rango de $700 \mathrm{a}$ $2000 \mathrm{~mm}$. La estación de lluvias es de junio a noviembre y una marcada estacionalidad, dividida en lluvias, "nortes" y secas (Orellana et al. 1999; Orellana et al. 2003). La temperatura promedio anual es de $26,3^{\circ} \mathrm{C}$. El estrato de vegetación dominante es la selva mediana subperennifolia en fase de sucesión (Martínez y Galindo-Leal 2002). Se presentan asociaciones de selva baja inundable, selva mediana subperennifolia, selva mediana subcaducifolia, sabanas, vegetación hidrófila y palmares de tasiste (Acoelorraphe wrightii) (Ocaña y Lot 1996; Martínez y Galindo-Leal 2002).

La mayoría de los habitantes hablan castellano y lenguas locales como el maya, chol y tzeltal. Las principales actividades productivas son la agricultura, ganadería y aprovechamiento del bosque. El sistema agrícola tradicional es la "roza-tumba-quema" para la siembra de maíz (Zea mays), frijol (Phaseolus vulgaris) y calabaza (Curcurbita pepo). La actividad ganadera consiste en sistemas de producción no tecnificados de bovinos, ovinos y caprinos. En el aprovechamiento del bosque se extrae madera, leña, palmas para techo de casas y plantas forrajeras. Otras actividades de importancia son la cacería para autoconsumo de pecaríes (Reyna-Hurtado et al. 2010; Briceño-Méndez et al. 2016), así como de otros mamíferos y aves mayores (Escamilla et al. 2000). La producción de miel en áreas forestales y en traspatios representa una fuente de ingreso para los habitantes locales, la cual es compatible con el ambiente y proporciona empleo a las familias (Parra 2016). También, es importante el aprovechamiento de la resina del árbol de chicozapote (Manilkara zapota) para elaborar chicle (Porter-Bolland et al. 2006).

\section{REGISTRO Y ANÁLISIS DE LA INFORMACIÓN}

Para el registró de información se seleccionaron 13 comunidades, 10 comunidades del municipio de Calakmul y tres del municipio de Hopelchen, de la zona de amortiguamiento de la Reserva de la Biosfera Calakmul, Campeche. Para caracterizar el sistema de producción de cerdos y pecaríes se aplicó un cuestionario, con entrevista directa a 169 campesinos criadores de cerdos en vida libre que se obtuvieron de un censo realizado en las 13 comunidades de estudio. Se registraron los siguientes datos: a) ubicación geográfica, distancia a la ANP (km), distancia a la carretera principal $(\mathrm{km})$, tierra total (ha) y tierra de cultivo (ha) en la unidad de producción; b) edad (años), escolaridad (años), integrantes de familia (número), experiencia en la crianza (años), personas que ayudan (número) y tiempo en el cuidado de los cerdos (horas/día) en los criadores de cerdos; c) total de cerdos y pecaríes (número), crías en desarrollo (número), cerdas de cría (número) y machos adultos (número); d) peso de lechones al destete $(\mathrm{kg})$, edad de pastoreo de lechones (meses), lechones por parto por cerda (número), mortalidad al destete (\%), número de partos por año y vida útil de la cerda (años); e) consumo mensual de grano maíz $(\mathrm{kg})$, consumo diario de forraje de plantas nativas $(\mathrm{kg})$ y costo de la alimentación (MXN\$) y f) venta anual 
de cerdos (número), peso a la venta $(\mathrm{kg})$, ingreso anual por ventas $(\mathrm{MXN} \$)$, venta anual de carne $(\mathrm{kg})$, ingreso anual de venta de lechones (MXN\$), ingreso de la renta del semental (MXN\$/año) y la distancia a los centros de consumo $(\mathrm{km})$. Las variables de consumo de alimentos, el peso vivo y los costos se determinaron con el productor y la ayuda del equipo de investigación. Adicionalmente, se registraron variables cualitativas como el gusto por la cría de cerdos (si, no), la crianza de otras especies de fauna silvestre $(1,2$, más de dos especies), la crianza de pecaríes (si, no), disponibilidad de mano de obra (1, 2, más de dos personas), disponibilidad de corrales para encierre (1,2, más de dos corrales), diversidad de alimentos ofrecidos (1, 2, más de dos), uso de forrajes en la dieta (nativo, cultivado, otro), autoconsumo de productos del cerdo (carne, productos procesados, otro) y la disponibilidad de ingresos (no, a veces, siempre).

La información de los cuestionarios se organizó en hojas de cálculo de Excel y se aplicó análisis de frecuencia, factorial, agrupación jerárquica y de varianza con el paquete estadístico SAS (SAS 2003). El análisis factorial, descrito por Khattree y Naik (2000), es un método de reducción de datos, lo hace buscando variables no observadas subyacentes (latentes) que se reflejan en las variables observadas (variables manifiestas). En donde, un factor es una variable hipotética que contribuye a explicar la varianza de al menos dos variables observadas y, al mismo tiempo, proporciona un resumen de las nuevas variables que se denominan factores y que son construidas, con las que se explicó la producción de cerdos en las localidades ubicadas en la Reserva de la Biósfera Calakmul. El nombre de cada uno de estos factores se determinó con base a su relación con las variables que más cargaron en el factor.

El objeto del análisis de agrupación jerárquica fue determinar la distancia entre los sistemas de crianza de cerdos con el procedimiento CLUSTER y el método AVERAGE del SAS (Muñoz, 2002). El análisis consistió en medir la distancia entre los sistemas de crianza de cerdos por la media de las unidades de producción de cada grupo. Para la descripción de los tres sistemas de crianza de cerdos se siguió la metodología de Usai et al (2006) y con un análisis de varianza se compararon las medias de los modelos probabilísticos utilizando el procedimiento GLM del SAS y la prueba de Tukey ajustada.

Para determinar la relación entre las tipologías de cerdos en vida libre con la crianza de pecaríes y otras especies de la fauna silvestre se utilizó el análisis de componentes principales cualitativos, con el uso de las variables cualitativas del cuestionario y el procedimiento PRINQUAL del SAS (Muñoz, 2002). El desarrollo matemático de los componentes cualitativos fue desarrollado por Kuhfeld et al. (1985), los resultados se presentan en una gráfica, en donde los sistemas de producción de cerdos son representadas como puntos y la estructura de las variables cualitativas de este estudio como vectores.

\section{RESULTADOS}

\section{FACTORES DE LA CRIANZA DE CERDOS}

La crianza del cerdo doméstico es parte del modo de vida de las familias y el pecarí de collar es criado en cautiverio a muy baja escala para el autoconsumo de carne o bien como una especie de ornato. Los siete componentes principales, con autovalor mayor que uno, explicaron 75\% de la varianza acumulada (Figura 1).

Los factores que determinaron la crianza de cerdos en vida libre en la Reserva de la Biósfera Calakmul fueron la eficiencia reproductiva, los insumos para la producción, los medios de producción y comercialización, el contexto socioeconómico, la compra de alimentos y el tamaño de la piara. Como se muestra en la Tabla I, aunque la crianza de cerdos en vida libre en la zona de estudio fue a pequeña escala, la mayor contribución para explicar la varianza de los datos fue la eficiencia reproductiva (Factor 1), que tuvo como variables con mayor carga en el factor al número de lechones por parto $(94 \%)$ y el número de partos por año (85\%). El principal insumo utilizado en la alimentación de los cerdos fue el grano de maíz (Factor II) y explicó $14,1 \%$ de la varianza de los datos. En los medios de producción y comercialización (Factor III), las variables con más carga en el factor fueron la distancia a la ANP (94\%), la disponibilidad de tierra (92\%) y la distancia a la carretera principal (87\%). El contexto socioeconómico (Factores IV y V) explicó el 16.7\% de la varianza de los datos, las variables con aporte a este factor fue la edad del productor (80\%), la experiencia en la cría de cerdos $(71 \%)$, la escolaridad $(76 \%)$ y el número de integrantes de la familia (67\%). El sexto factor explicó $5,5 \%$ de varianza de los datos y se relacionó con el consumo mensual de alimento $(72 \%)$ y la mortalidad de lechones al destete (69\%). Por último, el factor VII se le denominó tamaño de la piara, en donde el número total de cerdos (78\%) y el peso del cerdo a la venta (57\%) tuvieron la mayor correlación con este factor.

\section{SISTEMAS DE CRIANZA}

A continuación se describen los sistemas de crianza con los resultados de la frecuencia de las variables cualitativas, el análisis cluster, ANOVA y los componentes principales cualitativos. El análisis clúster unió a las unidades de producción de la crianza de cerdos en traspatio $(23,1 \%)$ con la crianza itinerante $(55 \%)$, en tanto, la itinerancia estacional $(21,9 \%)$ tuvo una variación promedio diferente a los otros sistemas.
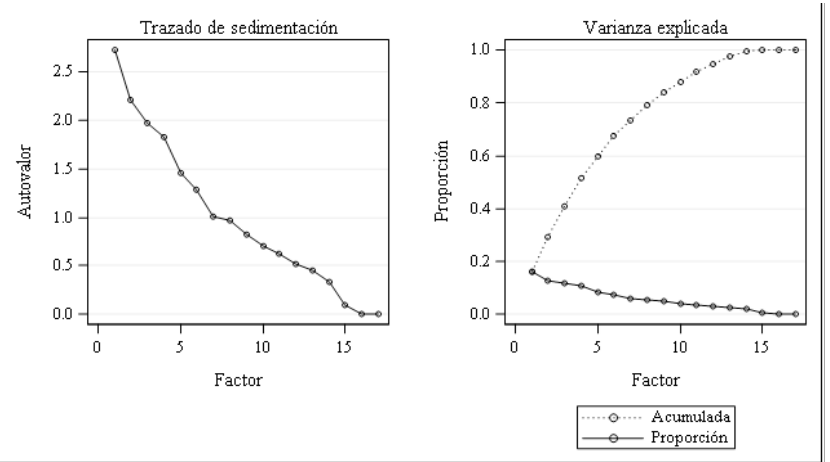

Figura 1. Componentes principales y proporción de varianza explicada en el análisis factorial para la crianza de cerdos en vida libre en el sureste de México (Analysis of principal components and proportion of variance explained in the factor analysis of free ranging pigs in the southeast of Mexico). 
Tabla I. Estructura factorial de la producción de cerdos en vida libre en el sureste de México (Factorial structure of the production of free ranging pigs in the southeastern Mexico).

\begin{tabular}{|c|c|c|c|c|c|c|c|}
\hline \multirow{2}{*}{ Variables } & \multicolumn{7}{|c|}{ Componentes } \\
\hline & 1 & II & III & IV & $\mathrm{V}$ & VI & VII \\
\hline \multicolumn{8}{|l|}{ Variables del productor y la unidad } \\
\hline Edad (años) & 13 & -1 & 10 & 80 & -6 & -5 & 8 \\
\hline Escolaridad (años) & -8 & -8 & 9 & -76 & 5 & 5 & 34 \\
\hline Número de integrantes de familia & 22 & 14 & 2 & 7 & 67 & 19 & -20 \\
\hline Experiencia en la crianza (años) & 9 & -2 & 13 & 71 & 19 & 6 & 15 \\
\hline Personas en el cuidado de cerdos (número) & 3 & 1 & 7 & -15 & 61 & 14 & 7 \\
\hline Distancia del hogar a la ANP (km) & 9 & 9 & 94 & 10 & 7 & 3 & -6 \\
\hline Distancia a la carretera $(\mathrm{km})$ & 21 & 5 & 87 & -1 & 8 & 11 & -5 \\
\hline Tierra total (ha) & 7 & 15 & 92 & 10 & 3 & 7 & 7 \\
\hline Tierra cultivada (ha) & 6 & 2 & 19 & 28 & 52 & -41 & 11 \\
\hline \multicolumn{8}{|l|}{ Variables reproductivas } \\
\hline Partos por cerda por año (número) & 85 & 6 & 20 & -3 & 5 & -7 & 5 \\
\hline Lechones por parto (número) & 94 & 3 & 1 & 5 & 6 & -2 & -2 \\
\hline Mortalidad al destete (\%) & 26 & -15 & 7 & -3 & 20 & 69 & -10 \\
\hline \multicolumn{8}{|l|}{ Variables de la piara } \\
\hline Cerdos por piara (número) & 28 & 15 & 7 & -3 & -15 & 9 & 78 \\
\hline Consumo mensual de alimento $(\mathrm{kg})$ & -16 & 3 & 15 & 3 & 7 & 72 & 17 \\
\hline $\begin{array}{l}\text { Consumo mensual de grano de } \\
\text { maíz }(\mathrm{kg})\end{array}$ & 4 & 98 & 5 & 2 & 4 & -1 & 6 \\
\hline Peso del cerdo a la venta $(\mathrm{kg})$ & -15 & 5 & -34 & 13 & 41 & -4 & 57 \\
\hline Varianza explicada & 3,38 & 2,93 & 2,81 & 1,87 & 1,44 & 1,30 & 1,22 \\
\hline Variación explicada (\%) & 21,6 & 14,1 & 11,7 & 9,2 & 7,5 & 5,5 & 5,2 \\
\hline Variación acumulada (\%) & 21,6 & 35,7 & 47,4 & 56,7 & 64,2 & 69,7 & 74,8 \\
\hline
\end{tabular}

Crianza en traspatio. En la crianza de traspatio los cerdos viven en el solar de las casas. En la Tabla II se muestra que los productores tienen el mayor número de integrantes de la familia, en tanto, la edad y escolaridad es similar con el sistema itinerante $(p<0,05)$. El tamaño de la piara es de 7,3 cerdos en total, y no hubo diferencia $(\mathrm{p}<0,05)$ con la agrupación de itinerancia estacional; tuvo dos cerdas adultas, 2,5 machos y el resto fueron lechones. En la reproducción, las cerdas tuvieron 6,3 lechones por parto, sin diferencia con la crianza itinerante $(\mathrm{p}<0,05)$. La mortalidad al destete $(8,6 \%)$ fue la más baja de los tres sistemas $(\mathrm{p}<0,05)$. Los costos de la alimentación (MXN\$8100,00) y el forrajes cosechado del bosque para la alimentación de los cerdos (5 kg/día) fueron más altos a los otros sistemas $(p<0,05)$. La venta de cerdos adultos se realizó a nivel regional y a una distancia de $11,5 \mathrm{~km}$ de la localidad. Cada unidad de producción vendió al año en promedio 1,7 cerdos adultos, $82,9 \mathrm{~kg}$ de carne y obtuvieron más ingresos (MXN\$3367,1). La crianza en traspatio tiene instalaciones para el resguardo de los cerdos, lo que se relaciona con una mayor frecuencia de uso de mano de obra y beneficio para los criadores (Figura 2). El análisis PRINQUAL, como se presenta en la Figura 3 , determinó que cuatro componentes principales tuvieron autovalor mayor a uno y explicaron $75 \%$ de la variación acumulada. La desviación a la derecha en el primer componente para la diversidad de alimentos ofrecidos en corral, el uso de forraje en la dieta y el gusto por la crianza de cerdos, representa una tendencia a la intensificación en el uso de insumos para el cuidado de los cerdos (Figura 4).

Crianza en itinerancia estacional. En la itinerancia estacional los cerdos permanecen en el solar de la casa y se dejan en libertad cuando no hay crecimiento de cultivos o cuando los granos de las cosechas escasean. La Tabla II muestra que los productores tienen menos edad (38,3 años), mayor escolaridad (7,8 años) y menor superficie de tierra de cultivo (2,8 ha), comparado con las otras agrupaciones $(\mathrm{p}<0,05)$. La piara tuvo 7,5 cerdos, sólo diferente con la crianza itinerante $(\mathrm{p}<0,05)$ y tuvieron el mayor número de lechones por parto $(7,2 \pm 0,2)$. La tasa de mortalidad al destete fue la más alta $(20 \%)$, con escasa medidas sanitarias preventivas y sin asistencia técnica. En la alimentación se ocupó la cantidad mensual más baja de grano de maíz (60,9 $\mathrm{kg} / \mathrm{mes}$ ) y un menor costo por año para este concepto (MXN\$2977,3/año). El consumo diario de forraje de

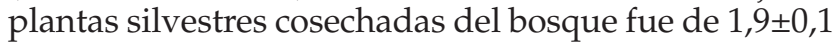
$\mathrm{kg}$ por cerdo, sin diferencia con la crianza itinerante. El costo de agua es menor (MXN\$206,0) en comparación a los otros sistemas de crianza $(p<0,05)$. La asistencia 
Tabla II. Medias mínimo cuadráticas de las variables de los sistemas de crianza de cerdos en vida libre en el sureste de México (Means obtained by the method of least squares for the variables of the groups of free ranging pigs in the southeast of Mexico).

Variables

Información del productor y la unidad

Edad (años)

Escolaridad (años)

Tiempo en la cría de cerdos (años)

Personas que apoyan la cría de cerdos

Tiempo destinado a la cría de cerdos (hr/día)

Integrantes de familia

Tierra total (ha)

Tierra de cultivo (ha)

Distancia a la ANP $(\mathrm{km})$

Distancia a carretera $(\mathrm{km})$

Piara de cerdos

Número de cerdos por piara

Número de cerdas adultas

Número de machos adultos

Peso al destete $(\mathrm{kg})$

Edad al pastoreo (meses)

Parámetros reproductivos

Lechones por parto (número)

Número de partos por año

Vida útil de la cerda (años)

Mortalidad al destete (\%)

Alimentación

Consumo mensual de grano maíz ( $\mathrm{kg} / \mathrm{mes}$ )

Consumo de forraje de plantas silvestres (kg/día)

Costo de alimentación (MXN\$/año)

Ventas e ingresos

Venta anual de cerdos (número)

Peso a la venta $(\mathrm{kg})$

Ingreso anual por ventas (MXN\$)

Venta anual de carne $(\mathrm{kg})$

Ingreso anual por venta de lechones (MXN\$)

Distancia de venta $(\mathrm{km})$

Ingreso anual por renta de semental (MXN\$/año)

$47,2 \pm 1,9^{\mathrm{a}}$
$6,0 \pm 0,4^{\mathrm{b}}$
$15,9 \pm 1,6$
$2,5 \pm 0,1$
$1,7 \pm 0,1$
$5,5 \pm 0,3^{\mathrm{a}}$
$131 \pm 15,7$
$3,6 \pm 0,3^{\mathrm{a}}$
$9,7 \pm 0,80$
$3,1 \pm 0,7^{\mathrm{ab}}$
$7,3 \pm 0,6^{\mathrm{a}}$
$1,9 \pm 0,2^{\mathrm{a}}$
$2,4 \pm 0,2^{\mathrm{a}}$
$5,8 \pm 0,2$
$1,2 \pm 0,1$

$6,3 \pm 0,2^{\mathrm{ab}}$
$1,5 \pm 0,1$
$2,9 \pm 0,1$
8,6

$150 \pm 4,6^{a}$

$5 \pm 0,2^{\mathrm{a}}$

$8100 \pm 243,8^{a}$

$1,7 \pm 0,1^{\mathrm{a}}$
$75,1 \pm 1,5$
$3367,1 \pm 231,4^{\mathrm{a}}$
$82,9 \pm 1,0^{\mathrm{b}}$
$313,7 \pm 49,8$
$11,5 \pm 1,2$
$287,6 \pm 16,0^{\text {ba }}$

$38,3 \pm 2,0^{b}$

$7,8 \pm 0,04^{a}$

$12 \pm 1,7$

$2,4 \pm 0,1$

$1,8 \pm 0,1$

$4,9 \pm 0,3^{\text {ba }}$

$123,4 \pm 16,1$

$2,8 \pm 0,3^{b}$

$8,9 \pm 0,8$

$4,00 \pm 0,7^{a}$

$7,5 \pm 0,6^{a}$

$1,5 \pm 0,0^{\text {ba }}$

$1,9 \pm 0,2^{\text {ab }}$

$6,6 \pm 0,2$

$1,3 \pm 0,1$

$7,2 \pm 0,2^{\mathrm{a}}$

$1,6 \pm 0,1$

$3,1 \pm 0,1$

20,0

$60,9 \pm 4,7^{b}$

$1,9 \pm 0,1^{\mathrm{b}}$

$2977,3 \pm 260,5^{b}$

$63,6 \pm 3,0^{\mathrm{b}}$

$1,9 \pm 0,1^{b}$

$3048,4 \pm 164,3^{b}$

$1,5 \pm 0,1^{\mathrm{ab}}$

$73,8 \pm 1,0$

$2945,8 \pm 1523^{\text {ba }}$

$84,5 \pm 0,6^{b}$

$371,7 \pm 25,5$

$12,5 \pm 0,8$

$270 \pm 10,1^{\mathrm{b}}$

a,b,c Medias de hilera con distinta letra tienen diferencia significativa $(p<0,05), \mathrm{n}=$ número de observaciones

técnica y el uso de medidas preventivas son escasas, $15,4 \%$ de los campesinos únicamente desparasitan a sus cerdos. El peso de venta de los cerdos fue de $71,1 \mathrm{~kg}$ e ingreso total de MXN\$2467,8 por año. Los productores vendieron menos cerdos finalizados $(1,3)$ y obtuvieron ingreso anual por la renta de semental (MXN\$325 $\pm 16,0)$. Con los resultados PRINQUAL, la itinerancia estacional y la crianza en traspatio mostraron una tendencia a la diversificación del alimento ofrecido y al uso de forrajes (Figura 4). Este sistema, al igual que en el de traspatio, no tuvo relación con la crianza de pecaríes y la fauna silvestre en cautiverio (Figura 4).

Crianza itinerante. La crianza itinerante de cerdos fue el sistema tradicional utilizado por los productores de mayor edad (49,5 años), con baja escolaridad (5,3 años) y menos integrantes de familia $(4,3)$. Los productores tienen un tamaño de piara de 4,2 cerdos y 1,1 cerdas adultas (Tabla II). El consumo diario de plantas silvestres por cerdo es de $1,9 \mathrm{~kg}$ y $63 \mathrm{~kg}$ de maíz por mes. El costo anual de la alimentación es intermedio (MXN\$3048,4) y no tuvo diferencia con la itinerancia 


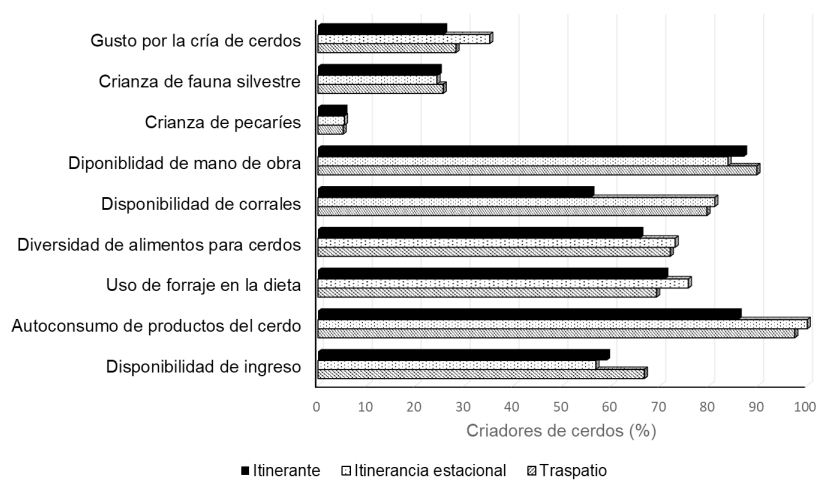

Figura 2. Frecuencia de las variables cualitativas de la crianza de cerdos en vida libre en el sureste de México (Frequency of qualitative variables of free ranging pigs in the southeast of Mexico).
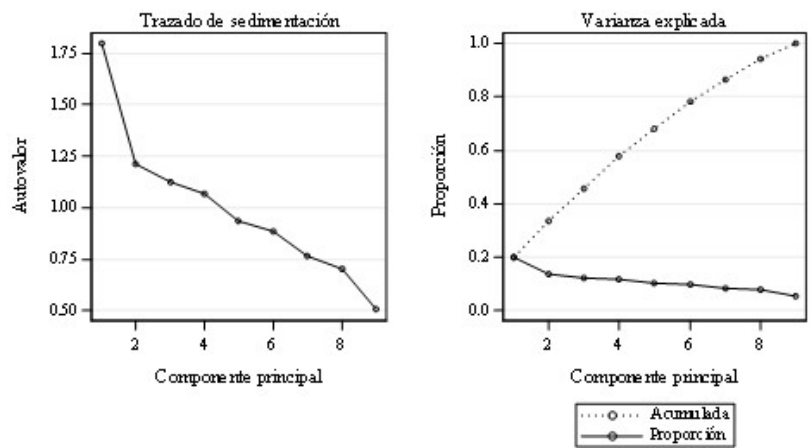

Figura 3. Autovalores y proporción de varianza explicada del análisis PRINQUAL en la crianza de cerdos en vida libre en el sureste de México (Eigenvalues and proportion of variance explained in the PRINQUAL analysis in the raising of pigs in free life in the southeast of Mexico).

estacional $(p<0,05)$. La venta de cerdos finalizados fue menor que en la crianza en traspatio, pero mayor a la itinerancia estacional $(p<0,05)$. La utilidad por renta de semental es menor comparado a los otros sistemas $(\mathrm{p}<0,05)$. Como resultados del análisis de componentes principales cualitativos, la crianza itinerante se asoció con el uso de la mano de obra para el manejo de los cerdos, la cría de pecarí de collar y otras especies de fauna silvestre en cautiverio (Figura 4). El uso de corrales de manejo, el autoconsumo de los productos del cerdo y los ingresos tuvieron menos ajuste a un modelo bidimensional (Figura 4).

\section{DISCUSIÓN}

La crianza de cerdos en vida libre es parte de una forma tradicional de producción en el área de estudio y contribuye a cubrir la demanda de carne para la alimentación de la familia y el aporte de ingresos económicos. La función social de la cría de cerdos fue ampliamente discutida por Kagira et al. (2010), Alawneh et al. (2014) y Leslie et al. (2015) en otros países, concluyendo que las familias producen cerdos en pie, carne para eventos socioculturales, productos transformados y cerdos para intercambio o regalo, resultados similares se encontraron en este estudio. Los medios de producción fueron la piara de cerdos criollos, menos de 10 cerdos, los alimentos y los corrales construidos con materiales de la región, que es común en la cría de cerdos en áreas marginadas (Rodríguez-Estévez et al. 2009; Dione et al. 2014; Leslie et al. 2015). La venta de cerdos se destinó al mercado local y regional, como fue registrado para otras regiones por Lemke y Valle-Zárate (2008), quienes establecieron que en las localidades cercanas a las zonas urbanas la producción de carne de cerdo es impulsada por la demanda del mercado a nivel local y regional, en tanto que, para las localidades alejadas están orientada a satisfacer los mercados informales o para autoconsumo.

El análisis factorial determinó que la varianza de los datos fue explicada por la eficiencia reproductiva, el nivel de uso de insumos para la producción y los beneficios que las familias obtienen de la crianza de cerdos. Las tipologías de crianza de cerdos en vida libre fueron de pequeña escala con diferencias en el uso de insumos, infraestructura, medios de producción y comercialización. El sistema más tradicional fue la crianza itinerante, clasificado como transitorio por Thys et al. (2016) en Zambia. Los tres sistemas son de tipo marginal y son adaptaciones a las áreas de bosques tropicales locales, como se ha mencionado para otras regiones (Dione et al. 2014; Leslie et al. 2015). El nivel de crianza en libertad de los cerdos estuvo definido por la tradición de la crianza sin ningún manejo, la cantidad de mano de obra, la disponibilidad de recursos económicos para infraestructura y la compra

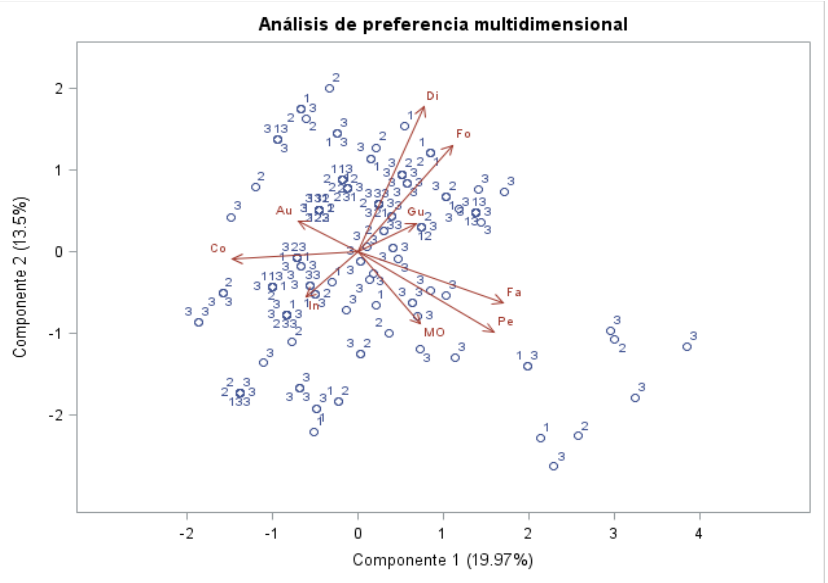

Figura 4. Representación bidimensional de las variables cualitativas (vectores) y los sistemas de crianza de cerdos en vida libre (puntos) en los dos primeros componentes principales generados con el análisis PRINQUAL del SAS en el sureste de México. Leyenda: Crianza en traspatio (1), itinerancia estacional (2), itinerante (3), Gu=Gusto por la crianza de cerdos, $\mathrm{MO}=$ disponibilidad de mano de obra, $\mathrm{Di}=$ Diversidad de alimentos ofrecidos, Fo=Uso de forrajes, $\mathrm{Fa}=$ cría de cerdos y fauna silvestre, $\mathrm{Pe}=$ cría de cerdos y pecarí, In= ingresos, $\mathrm{Co}=$ infraestructura, $\mathrm{Au}=$ autoconsumo de carne (Bi-plot of qualitative variables with free ranging pigs in the southeastern Mexico: Backyard pig raising (1), Seasonal roaming pigs (2), free roaming pigs (3), $\mathrm{Gu}=$ enjoy pigs raising, $\mathrm{MO}=$ labor availability, $\mathrm{Di}=$ pig feed diversity, $\mathrm{Fo}=$ Forages use, $\mathrm{Fa}=\mathrm{Pig}$ and wildlife raising, $\mathrm{Pe}=$ pigs and peccary raising, $\mathrm{In}=$ income, $\mathrm{Co}=$ pig pens, $\mathrm{Au}=$ meat consumption) 
de alimentos, como lo registraron Kagira et al. (2010) en Kenia. La producción en traspatio y en itinerancia estacional se realizó por los productores de menos edad y disponibilidad de mano de obra. Los insumos para la alimentación fueron grano de maíz, complementado con desperdicios de cocina, frutos y plantas silvestres, como fue descrito en otros estudios (Rodríguez-Canul et al. 1998; Copado et al. 2004; Martínez et al. 2016). En la alimentación en corral de los cerdos en traspatio e itinerancia estacional se utilizó forraje de corte de árboles silvestres como el ramón (Brosimum alicastrum), huachim (Leucaena leucocephala), el guácimo (Guazuma umbilifolia) y el ciruelo silvestre (Spondias spp), así como gramíneas (Brachiaria brizantha y Saccharum officinarum). Estos resultados coinciden en parte con Leluke y Kyvsgaard (2003), quienes encontraron que los productores en las zonas rurales de África y del sur de Asia, alimentan a sus cerdos principalmente con plantas forrajeras, subproductos de cultivos agrícolas y restos de alimentos de cocina.

En el caso del pecarí de collar, éste estuvo presente en los sistemas locales de producción de cerdos domésticos, es valorado por su aporte de carne para las familias y en algunos casos es criado como animal de ornato. En la zona de estudio la crianza de pecaríes no representó un activo en los medios de vida de las familias a diferencia del cerdo doméstico, aunque su crianza en cautiverio es una alternativa para consumir su carne y para que los campesinos dejen de cazarlos en las áreas naturales protegidas.

Considerando el planteamiento del Programa de Manejo de la Reserva de la Biósfera Calakmul, de recuperar y proteger la diversidad biológica con el establecimiento de criaderos con especies silvestres y de fácil manejo, fueron los productores de la crianza itinerante los que tuvieron experiencia para adoptar la crianza de pecaríes silvestres, por no orientar la producción al mercado, sino más bien lo que buscan es producir carne para autoconsumo y ocasionalmente la venta. Este tipo de productor carece de instalaciones para la crianza de cerdos, pero generalmente cuando se promueve un proyecto de conservación participativo las actividades se acompañan de estímulos a la producción, los cuales deben de incluir infraestructura y asistencia técnica para el manejo de las especies de fauna silvestre.

El control de la crianza del cerdo doméstico en la Reserva de la Biósfera Calakmul no está determinada por la normatividad ambiental y la amenaza que representa esta especie para los ecosistemas, sino más bien está restringida por los reglamentos internos de las comunidades. Una de las razones principales para la restricción de la crianza de cerdos en vida libre es para proteger las fuentes de agua, los cultivos agrícolas, las plantaciones forestales y los huertos de traspatios. También, la crianza de los cerdos en vida libre ha estado sometida a diferentes presiones para erradicarlas de las comunidades rurales, en donde la itinerancia de los cerdos se considera un proceso insalubre (Thys et al. 2016). Uno de los motivos para el encierre del cerdo es evitar el consumo de excretas humanas y de otros animales, aunque esto está más relacionado con la cultura de los pueblos por defecar al aire libre. Otro as- pecto que puede influir para sugerir el confinamiento de los cerdos es la presencia de enfermedades, cuando un cerdo anda libre tiene contacto directo con animales domésticos y silvestres, lo que favorece el contagio, sobre todo en regiones donde se desconocen las medidas de medicina preventiva (FAO 2010). Sin embargo, en la parte ambiental, cuando se considera la relación hombre-cría de cerdos-ecosistema para el desarrollo sustentable de las comunidades rurales, a los cerdos se les ve como el medio para vincular la conservación con la biodiversidad (Thys et al. 2016); para el caso del estudio, los productores tuvieron el conocimiento para utilizar las plantas forrajeras y frutos silvestres (bellotas) para la alimentación de cerdos, como se ha mencionado en otros estudios (Porras et al. 1997). Esto representa una oportunidad para promover la producción de cerdo orgánico y orientar la producción a un ciclo bien definido para tener acceso al mercado, además de responder a las necesidades y a las costumbres de los pueblos.

\section{CONCLUSIONES}

En la Reserva de la Biósfera Calakmul en el sureste de México, el cerdo doméstico es un activo de las estrategias adaptativas de modos de vida de los campesinos, en tanto el pecarí de collar es una especie de ornato o para el autoconsumo ocasional de carne. El sistema de producción itinerante de cerdos fue el más común en la región de estudio, en tanto la crianza en traspatio y en itinerancia estacional está en transición a un sistema de confinamiento. La mayor convivencia del cerdo doméstico con el pecarí de collar se tuvo en la crianza itinerante. El propósito principal de la producción del cerdo fue como ahorro para cubrir las emergencias económicas, satisfacer las demandas familiares de carne y de productos procesados. Las prácticas de alimentación precarias, medicina preventiva y la nula asistencia técnica en los cerdos domésticos representaron una oportunidad para incorporar especies de pecaríes silvestres, con menos exigencias en el manejo, a los sistemas locales de producción. Los reglamentos internos de las comunidades restringen la cría de cerdos en vida libre en el área de estudio y no el Plan de Manejo de la Reserva de la Biósfera Calakmul. De seguir las recomendaciones del Plan de Manejo de sustituir la crianza de animales domésticos por especies de fauna silvestre, en este caso la cría en cautiverio de pecaríes, será una alternativa posible para evitar daños a los ecosistemas. Es necesario continuar con investigaciones para incorporar el uso de productos del bosque para darle un valor agregado a la carne de cerdos criados en confinamiento y así evitar daños a los ecosistemas naturales. Para estructurar la producción de cerdos o pecarí de collar en las comunidades rurales se debe integrar a los pequeños productores en sociedades o cooperativas locales, que les permitan ingresar a un mercado justo.

\section{AGRADECIMIENTOS}

Al Consejo Nacional de Ciencia y Tecnología por la beca de Posgrado (No.162386). Al Colegio de Postgraduados, Campus Puebla y la beca Russell E. Train 
WWF-EFN (Acuerdo RM755) por el apoyo financiero para el trabajo de campo. A los productores de Campeche que colaboraron de manera voluntaria con las entrevistas. A Leonardo Pretelt Quintero y Macarena González Abarzúa, estudiantes de Posgrado en Ciencias Veterinarias Tropicales de la Universidad Nacional, Costa Rica, por la ayuda en la colecta datos de campo.

\section{BIBLIOGRAFÍA}

Ahmed, ST, Hong-Seok, M, Islam, MM, Seok-Young, K \& Chul-Ju, Y 2016, 'Effects of dietary natural \& fermented herb combination on growth performance, carcass traits and meat quality in grower-finisher pigs', Meat Science, vol. 122, pp. 7-15

Alawneh, Jl, Barnes, TS, Parke, C, Lapuz, E, David, E, Basinang, V, Baluyut, A, Villar, E, Lopez, EL \& Blackhall, PJ 2014, 'Description of the pig production systems, biosecurity practices and herd health providers in two provinces with high swine density in the Philippines', Preventive Veterinary Medicine, vol. 114, pp. 73-87.

Briceño-Méndez, M, Naranjo, JE, Mandujano, S, Altrichter, M \& ReynaHurtado, R 2016, 'Responses of two sympatric species of peccaries (Tayassu pecari and Pecari tajacu) to hunting in Calakmul, Mexico', Tropical Conservation Science, vol. 9, no. 3. pp. 1-11.

Borges-Ventura, DI, Montes-Pérez, R, Sarmiento-Franco, L \& SolorioSánchez, F 2014, 'Efecto de la suplementación de ensilado de pasto taiwán (Pennisetum purpureum) y ramón (Brosimum alicastrum) sobre el cambio de peso corporal y variables hemáticas del pecarí de collar (Pecarítajacu)en cautiverio', Tropical and Subtropical Agroecosystems, vol. 17, pp. 277-279.

Copado, F, de Aluja, AS, Mayagoitia, L \& Galindo, F 2004, 'The behaviour of free ranging pigs in the Mexican tropics and its relationships with human faeces consumption', Applied Animal Behaviour Science, vol. 88, pp. 243-252.

Dione, MM, Ouma, EA, Roesel, K, Kungu, J, Lule, P \& Pezo, D 2014, 'Participatory assessment of animal health and husbandry practices in smallholder pig production systems in three high poverty districts in Uganda', Preventive Veterinary Medicine, vol. 117, pp. 565-576.

Edwards, SA 2005, 'Product quality attributes associated with outdoor pig production', Livestock Production Science, vol. 94, no. 1, pp. 5-14.

Escamilla, A, Sanvicente, LM, Sosa, M \& Galindo-Leal, C 2000, 'Habitat mosaic, wildlife availability and hunting in the tropical forest of Calakmul, Mexico', Conservation Biology, vol. 14, no. 6, pp. 1592-1601.

FAO 2010, Good practices for biosecurity in the pig sector: Issues and options in developing and transition countries, FAO Animal Production and Health Paper, no 169, Rome, FAO.

Hadjikoumis, A 2012, 'Traditional pig herding practices in southwest Iberia: Questions of scale and zooarchaeological implications', Journal of Anthropological Archaeology, vol. 31, pp. 353-364.

Instituto Nacional de Ecología 2000, Programa de Manejo de la Reserva de la Biósfera Calakmul.

Jori, F, Gama, SLG, Mendes, A \& Siqueira, CNS 2004, 'El manejo colectivo de grandes grupos de Pecarí de collar (Tayassu tajacu) nacidos en cautividad. Un paso hacia el "ranching"?", ponencia presentada en el Congreso de Manejo de Fauna silvestre en Amazonia y Latinoamérica, agosto 2004

Kagira, JM, Kanyari, PWN, Maingi, N, Githigia, SM, Ng'ang'a, JC \& Karuga, JW 2010, 'Characteristics of the smallholder free-range pig production system in western Kenya', Tropical Animal Health Production, vol. 42, pp. 865-873

Karki, ST 2013, 'Do protected areas and conservation incentives contribute to sustainable livelihoods? A case study of Bardia National Park, Nepal', Journal of Environmental Management, vol. 128, pp. 988-999.

Khattree, R \& Naik, DN 2000, 'Multivariate data reduction and discrimination with SAS software, Cary, N.C., USA, SAS Institute Inc.
Kiltie, RA 1981, 'Stomach contents of rain forest peccaries (Tayassu tajacu and T. pecari)', Biotropica, vol. 13, pp. 234-236.

Kuhfeld, W. F., W. S. Sarle y F. W. Young. 1985. Methods of generating model estimates in the PRINQUAL macro. In: SUGI 10: proceedings of the 10th annual SAS users group international conference on SAS users group international conference Reno, Nevada, USA. pp. 962971. Consultado mayo 2018.

Leluke, FP \& Kyvsgaard, NC 2003, 'Improving pig husbandry in tropical resource-poor communities and its potential to reduce risk of porcine cysticercosis', Acta Tropica, vol. 87, pp. 111-117.

Lemke, U, Kaufmann, B, Thuy, LT, Emrich, K \& Valle-Zárate, A 2006, 'Evaluation of smallholder pig production systems in North Vietnam: Pig production management and pig performances', Livestock Science, vol. 105, pp. 229-243.

Lemke, U \& Valle-Zárate, A 2008, 'Dynamics and developmental trends of smallholder pig production systems in North Vietnam', Agricultural Systems, vol. 96, pp. 207-223.

Leslie, EEC, Geon, M, Abdurrahman, M, Ward, MP \& Toribio, LMLA 2015, 'A description a smallholder pig production systems in eastern Indonesia', Preventive Veterinary Medicine, vol. 188, pp. 319-327.

Levin, MJ \& Ayres, WS 2017, 'Managed agroforests, swiddening, and the introduction of pigs in Pohnpei, Micronesia: Phytolith evidence from an anthropogenic landscape', Quaternary International, vol. pp. 434: 70-77.

Manson, S 2006, 'Land use in the southern Yucatán peninsular region of Mexico: Scenarios of population and institutional change', Computers, Environment \& Urban Systems, vol. 30, pp. 230-253.

Martínez, E \& Galindo-Leal, C 2002, 'La vegetación de Calakmul, Campeche, México. Clasificación, descripción y distribución', Boletín de la Sociedad Botánica de México, vol. 71, pp. 7-32.

Martínez, VG, Román, PSI, Vélez, IA, Cabrera, TE, Cantú, CA, de la Cruz, CL, Durán, AM, Maldonado, JJA, Martínez, SFE, Ríos, UA, Vega, MVE \& Ruiz, LFJ 2016, 'Morfometría del cerdo de traspatio en áreas rurales de México', Revista Mexicana de Ciencias Pecuarias, vol. 7, pp. 431-440.

Muñoz, SA 2002, Estadística aplicada uni y multivariante. Tomo II. Junta de Andalucía, Consejería de Agricultura y Pesca. Sevilla, España, pp. 756-757.

Navarrete, ZM, Lí, EO, Montoya, GE \& Gálvez CH 2000, 'Estudio hematológico comparativo del sajino (Tayassu tajacu) criado en cautiverio en Lima e lquitos', Revista de Investigaciones Veterinarias del Perú, vol. 11, pp. 170-172

Ocaña, D \& Lot, A 1996, 'Estudio de la vegetación acuática vascular del sistema fluvio-lagunar deltaico del río Palizada, Campeche, México', Anales del Instituto de Biología, Serie Botánica, vol. 67, pp. 303-327 Orellana, R, Balam, My Bañuelos, I (Coord.). 1999, 'Evaluación climática (Climatología de la Península de Yucatán)', In García de Fuentes, A, Córdoba, C, Ordoñez, P \& Ponce de León, P (ed.), Atlas de procesos territoriales de Yucatán. Facultad de Arquitectura, Universidad Autónoma de Yucatán, Mérida, México, pp. 194.

Orellana, R, Islebe, G \& Espadas, C 2003,' Presente, pasado y futuro de los climas de la Península de Yucatán', In Colunga-García, MP \& Larqué-Saavedra, A (ed.), Naturaleza y sociedad en el área Maya. Pasado, presente y futuro, Academia Mexicana de Ciencias. México., 52 pp. https://www.researchgate.net/publication/281850731_Presente_pasado_y_futuro_de_los_climas_de_la_Peninsula_de_Yucatan. Parra, AC 2016, 'Instrumento rector apícola para el Municipio de Calakmul, Campeche', Informe final, Deutsche Gesellschaft für Internationale Zusammenarbeit (GIZ) y Comisión Nacional de Áreas Naturales Protegidas, pp. 40 pp. http://selvamaya.info/wp-content/ uploads/2016/06/Instrumento-Rector-Ap\%C3\%ADcola-del-Municipio-de-Calakmul-Campeche.pdf 
Phengsavanh, P, Ogle, B, Stür, W, Frankow-Lindberg, BE \& Lindberg, JE 2011 , 'Smallholder pig rearing systems in northern Lao PDR', AsianAustralian Journal Animal Science, vol. 24: pp. 867-874.

Porras, TC, Martínez, R \& Fernández, R 1997, 'Sistemas agrarios tradicionales de dehesa en las comarcas de la Sierra y los Andévalos de la Provincia de Huelva: proyecto interregional: estudio de sistemas agrarios tradicionales en Andalucía, Algarve y Alentejo', Junta de Andalucía, Consejería de Agricultura y Pesca. pp. 313.

Porter-Bolland, LP, Drew, AP \& Vergara-Tenorio, C 2006, 'Analysis of a natural resources management system in the Calakmul Biosphere Reserve', Landscape \& Urban Planning, vol. 74, pp. 223-241.

Reyna-Hurtado, R, Naranjo, JE, Chapman, AC \& Tanner, GW 2010, 'Hunting and the conservation of a social ungulate: the white-lipped peccary Tayassu pecari in the Calakmul, Mexico', Oryx, vol. 44, pp. 88-96.

Reynolds, AM, Ropert-Coudert, Y, Kato, A, Chiaradia, A \& Maclntosh, AJ 2015, 'A priority-based queuing process explanation for scalefree foraging behaviours', Animal Behaviour, vol. 108, pp. 67-71.

Rodríguez-Canul, R, Allan, JC, Domínguez, JL, Villegas, S, Cob, L, Rodríguez, L, Cook, AJ, Williams, J, Argaez, F\& Craig, PS 1998, 'Application of an immunoassay to determine risk factors associated with porcine cysticercosis in rural areas of Yucatan, Mexico', Veterinary Parasitology, vol. 79, pp. 165-180.
Rodríguez-Estévez, V, García, A, Peña, F \& Gómez, AG 2009, 'Foraging of Iberian fattening pigs grazing natural pasture in the dehesa', Livestock Science, vol. 120, pp. 135-143.

Rosenthal, JS 2010, 'Are view of the role of protected areas in conserving global domestic animal diversity', Animal Genetic Resources, vol. 47, pp. 101-113. Ruiz-Mallén, I, Corbera, E, Calvo-Boyero, D \& ReyesGarcía, V 2015, 'Participatory scenarios to explore local adaptation to global change in biosphere reserves: Experiences from Bolivia and Mexico', Environmental Science \& Policy, vol. 54, pp. 398-408.

Statistical Analysis Systems (SAS), 2003, The analyst application, Second ed. Cary, NC, SAS Inst Inc.

Thomas, LF, de Glanville, WA, Cook, EA \& Fèvre, EM 2013, 'The spatial ecology of free-ranging domestic pigs (Sus scrofa) in western Kenya', Veterinary Research, vol. 9, pp. 2-12.

Thys, S, Mwape, KE, Lefevre, P, Dorny, P, Phiri, AM, Marcotty, T, Phiri, IK \& Gabriël, S 2016, 'Why pigs are free-roaming: Communities' perceptions, knowledge and practices regarding pig management and taeniosis/cysticercosis in a Taenia solium endemic rural area in Eastern Zambia', Veterinary Parasitology, vol. 225, pp. 33-42.

Usai, MG, Casu, S, Molle, G, Decandia, M, Ligios, S \& Carta, A 2006, Using cluster analysis to characterize the goat farming systems in Sardinia', Livestock Science, vol. 104, pp. 63-67. 